\title{
Coexistence of choline acetyltransferase and GABA in axon terminals in the dorsal cap of the rat inferior olive
}

\author{
A.R. Caffé, R.K. Hawkins, C.I. De Zeeuw * \\ Department of Anatomy, Erasmus University Rotterdam. PO Box 1738, 3000 DR Rotterdam, The Netherlands
}

Accepted 27 February 1996

\begin{abstract}
The dorsal cap of Kooy of the inferior olive (DC) is involved in compensatory eye movements. The rat DC receives a prominent input from the nucleus prepositus hypoglossi (NPH); part of these axon terminals are immunoreactive for choline acetyltransferase (ChAT) and part of them are GABAergic. In the present study we investigated the fine distribution of cholinergic terminals in the rat DC, and the possible coexistence of ChAT and GABA. ChAT-positive terminals were observed throughout the entire neuropil of the rat DC contacting both extraglomerular and intraglomerular dendrites. Twenty nine percent of these terminals also contained GABA. The ChAT/GABA double-labelled terminals showed the same morphological characteristics as terminals traced from the NPH. The present data demonstrate colocalization of ChAT and GABA in axon terminals of the rat DC and strongly suggest that neurons in the NPH are the source of these profiles.
\end{abstract}

Keywords: Acetylcholine; $\gamma$-Aminobutyric acid; Nucleus prepositus hypoglossi; Optokinetic reflex; Electron microscopy; Crest synapse

The dorsal cap of Kooy (DC) and the ventrolateral outgrowth (VLO) of the inferior olive are part of the anatomical circuit involved in the optokinetic reflex (e.g. $[1,3,12,20])$. Direction selective optokinetic signals from the retinal ganglion cells reach the DC and VLO via different tectal and tegmental nuclei. The caudal DC receives its input predominantly from the nucleus of the optic tract and is primarily involved in horizontal eye movements. The rostral DC and VLO receive their input from the terminal nuclei of the accessory optic tract and the ventral tegmental relay zone, and are involved in vertical eye movements $[11,14,15]$. The DC and VLO, in turn, send the optokinetic information via climbing fibres to the contralateral vestibulocerebellum $[5,20,22]$.

Studies in the rabbit have indicated that the descending pathways mentioned above are non-GABAergic and presumably excitatory [18]. In addition, the DC and VLO receive a prominent GABAergic input; the caudal DC receives most of its GABAergic input from the nucleus prepositus hypoglossi (NPH) [10] while the rostral DC and VLO receive their GABAergic innervation predominantly from the ventral dendate nucleus and dorsal group y [8].

\footnotetext{
* Corresponding author. Fax: (31) (10) 4365780.
}

GABAergic terminals in the DC and VLO mostly have pleomorphic vesicles and symmetric synapses and they are situated both in the extra- and intraglomerular neuropil. Within the latter location these terminals frequently contact dendrites linked by dendrodendritic gap junctions. The DC and VLO contain more crest synapses than any other olivary subnucleus; the vast majority of these terminals are GABAergic [8].

The DC also receives a dense cholinergic projection [4]. These cholinergic terminals, which are absent in the VLO, originate from the NPH and adjacent medial vestibular nucleus (MVN). The fact that the NPH provides both a GABAergic and a cholinergic projection to the DC raises the possibility that both neurotransmitters coexist within the same terminals. In the present study we investigated this possible colocalization and, in addition, we studied the ultrastructural characteristics of the terminals immunoreactive for choline acetyltransferase (ChAT) and compared them to those of the GABAergic terminals traced from the NPH. Since the rat DC has not yet been studied using this anti-ChAT antiserum we also report light microscopy observations.

Fourteen adult Wistar rats were used, five for light microscopy and nine for electron microscopy. For light microscopy, the animals were anaesthetized with Nembutal 
$(0.1 \mathrm{ml} / 100 \mathrm{~g} \mathrm{b.wt})$, and subsequently intracardially perfused with saline $(200 \mathrm{ml})$. Animals processed for ChAT were perfused with a fixative of $4 \%$ paraformaldehyde $(\mathrm{pH}$ 7.4) in $0.1 \mathrm{M}$ phosphate buffer, while animals processed for glutamic acid decarboxylase, the GABA synthesizing enzyme, were perfused with $0.5 \%$ zinc-salicylate and $4 \%$ paraformaldehyde $(\mathrm{pH} 4.5)$. The brains were removed and blocks containing the inferior olive were immersed for an additional $2 \mathrm{~h}$ in the same fixative. Sections of $40 \mu \mathrm{m}$ were cut on a cryotome, rinsed, and incubated in antisera against ChAT (Ab17; 1:500: kindly donated by Prof. C.
Cozzari, Rome, Italy [24]) or glutamic acid decarboxylase (1:2000; kindly provided by Prof. E. Mugnaini, Chicago, USA $[16,17,19])$. Binding of the primary antibodies was visualized with the avidin-biotin complex ( $A B C$ ) method, while diaminobenzidine (DAB) was employed as a chromogen. The labelled sections were washed, dehydrated, cleared in xylene, mounted on glass slides, and coverslipped.

For electron microscopy, two types of experiments were performed: (1) ChAT/GABA double labelling; and (2) anterograde tracing of wheat germ agglutininated
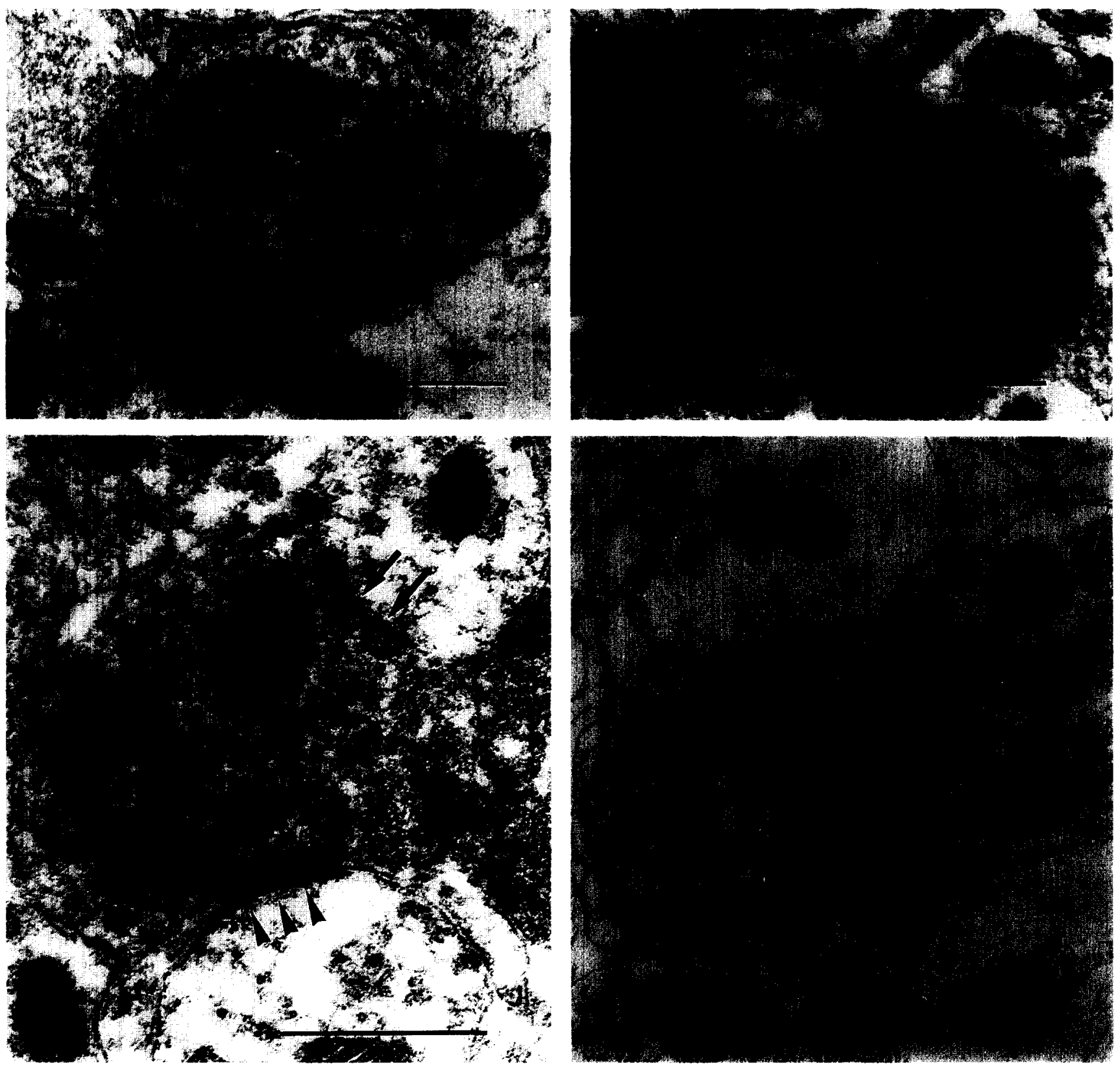

Fig. 1. Electron micrographs of the rat DC. (A) Terminal displaying immunoreactivity for both ChAT (DAB precipitate) and GABA (black dots indicate 15 $\mathrm{nm}$ gold particles). (B) Crest synapse formed by two cholinergic terminals; note the clear asymmetric synapses involved in the crest synapse. (C) Double ChAT/GABA-labelled terminal establishing both an asymmetric (arrows) and a symmetric (arrowheads) synapse with two different dendrites. (D) Double WGA-HRP/GABA-labelled terminal traced from the NPH that makes both an asymmetric (arrows) and a symmetric (arrowheads) synaptic contact with two different dendrites (large arrow indicates WGA-HRP reaction product). Note the similarity between the terminal in $\mathrm{C}$ and $\mathrm{D}$. Scale bars indicate $1 \mu \mathrm{m}$. 
horseradish peroxidase (WGA-HRP) from the NPH combined with GABA postembedding immunocytochemistry. For double labelling of ChAT and GABA the animals were anaesthetized with Nembutal $(0.1 \mathrm{ml} / 100 \mathrm{~g}$ b.wt $)$, perfused with saline, and subsequently fixed with $2 \%$ paraformaldehyde and $2 \%$ glutaraldehyde $(\mathrm{pH} \mathrm{7.3)}$ in 0.1 $\mathrm{M}$ cacodylate buffer. After a 2 -h postfixation period, 80 $\mu \mathrm{m}$ vibratome sections were cut and ChAT terminals were labelled using the $\mathrm{ABC}$ technique as described above. The parts containing stained terminals in the DC were dissected, osmicated in $1.5 \% \mathrm{OsO}_{4}$ overnight, stained en block in uranyl acetate, and embedded in plastic. From these tissue blocks, ultrathin sections were cut and incubated in an antiserum against GABA (1:1000, generously supplied by Prof. R.M. Buijs, Amsterdam, Netherlands $[6,21])$. The primary antiserum against GABA labelling was visualized with the use of $15 \mathrm{~nm}$ immunogold particles linked to a secondary antibody. Control staining was achieved by omitting the primary antibody from the solvent.

The second type of electron microscopic experiment was performed as published elsewhere [8-10]. In short, the animals were anaesthetized, injected with WGA-HRP in the NPH, and allowed to recover. After a survival period of two days, the animals were reanaesthetized with Nembutal $(0.1 \mathrm{ml} / 100 \mathrm{~g} \mathrm{b.wt})$ and intracardially perfused with saline and 5\% glutaraldehyde in $0.1 \mathrm{M}$ cacodylate buffer. Vibratome sections containing the DC were reacted with tetramethylbenzidine and stabilized with diaminobenzidine-cobalt. Subsequently, the sections with anterograde labelling were imbedded in plastic and processed for postembedding GABA immunocytochemistry as described above.

The light microscopic examination of the rat inferior olive showed a heavy cholinergic innervation, which was almost exclusively present in the DC. The $\beta$-nucleus and VLO showed only a few single fibres. In contrast, the entire rat olivary complex displayed GAD terminals. The highest degree of GAD innervation was observed in the $\beta$-nucleus. The DC and VLO contained a lower, but still appreciable, density of GABAergic boutons. These results do not differ from previous reports $[4,8,10,17,23]$.

The electron microscopic examination of the ultrathin sections of the rat DC showed four categories of terminals, i.e. single ChAT, single GABA, double-labelled, and unlabelled profiles (Fig. 1A,B,C); no cholinergic or GABAergic cell bodies or dendrites were observed. ChAT-labelled terminals were characterized by an electron-dense $\mathrm{DAB}$ precipitate (Fig. 1A,B,C). The DAB precipitate appeared as patchy densifications. This is the case even in the most optimal staining conditions, but in the illustrations presented here this feature is accentuated, because $2 \%$ glutaraldehyde had been added to the fixative. This glutaraldehyde is required for GABA labelling but hinders the ChAT immunostaining. Control stainings remained completely unlabelled. A terminal was classified as GABA-
Table 1

Percentages of single ChAT-immunoreactive, GABAergic, doublelabelled, and unlabelled axon terminals in intra- and extraglomerular locations in the rat $\mathrm{DC}$

\begin{tabular}{lllll}
\hline & ChAT & GABA & Double & Unlabelled \\
\hline$n=840$ & $\pm 27 \%$ & $\pm 11 \%$ & $\pm 11 \%$ & $\pm 51 \%$ \\
Extraglomerular & $\pm 76 \%$ & $\pm 81 \%$ & $\pm 75 \%$ & $\pm 88 \%$ \\
Intraglomerular & $\pm 24 \%$ & $\pm 19 \%$ & $\pm 25 \%$ & $\pm 12 \%$ \\
\hline
\end{tabular}

positive when it contained at least six times more gold particles than surrounding nonlabelled profiles with the same surface area. A total of 840 terminals was sampled in electron micrographs obtained from nonserial sections (for details of the sampling procedure see [9]). A summary of the quantitative data is given in Table 1. Two hundred and twenty-eight $(27.2 \%)$ of the axon terminals were single ChAT labelled. These terminal profiles were usually circular with a diameter of 0.8 to $1.8 \mu \mathrm{m}$. The ChAT-labelled terminals were filled with predominantly pleomorphic vesicles and an occasional dense core vesicle (Fig. 1B). The vast majority were apposed to dendritic profiles; only a few ChAT-labelled terminals contacted a cell body (data not shown). Most $(76 \%)$ of the terminals apposed to dendrites were located in the extraglomerular neuropil. They showed both asymmetric and symmetric synapses (Fig. 1B,C). Sometimes, the ChAT-labelled terminals formed crest synapses; these synapses are characterized by two asymmetric synapses contacting a thin dendritic element (Fig. 1B).

Ninety-one $(10.8 \%)$ of the sampled axon terminals were single GABA labelled (Table 1). The morphological characteristics and the postsynaptic distribution of these terminals were as described in detail in previous publications [8-10,23]. They contained mostly pleomorphic vesicles, established predominantly symmetric synapses, and were located also both in the intraglomerular and extraglomerular neuropil. Some of the GABAergic terminals inside the glomeruli were strategically located next to dendrites coupled by gap junctions, and some of the extraglomerular terminals formed crest synapses.

Ninety-four $(11.2 \%)$ of the sampled axon terminals were ChAT/GABA double labelled (Fig. 1A,C). The ChAT/GABA-positive axon terminals showed the general structural and spatial characteristics of the single ChATand GABA-labelled profiles described above. They were present inside $(25 \%)$ and outside $(75 \%)$ glomeruli and also formed asymmetric and symmetric synaptic complexes. Relatively frequently, individual terminals of this type formed an asymmetric as well as a symmetric synapse, each with a different postsynaptic dendrite (Fig. 1C).

Combined WGA-HRP anterograde tracing from the NPH and GABA immunostaining resulted in doublelabelled terminals in the DC (see also [10]). The singleand double-labelled terminals showed the same ultrastructural features as those of the cholinergic and GABAergic 
terminals. Interestingly, like the ChAT/GABA doublelabelled terminals, individual WGA-HRP/GABA doublelabelled terminals from the NPH were also observed to establish both symmetric and asymmetric synapses with different dendrites (Fig. 1D). We were unable to find double WGA/HRP-labelled terminals that formed crest synapses in the DC.

The main finding of the present study is the demonstration of colocalization of ChAT and GABA immunoreactivity in axon terminals of the rat DC. Because the NPH contains both cholinergic and GABAergic neurons $[4,10]$ and because the double ChAT/GABA-labelled terminals show the same morphological characteristics as the GABAergic terminals anterogradely labelled from the NPH, the NPH is the most likely source for the coexistence of these neurotransmitters in the DC. The function of this coexistence is unclear. The finding that individual double ChAT/GABA-labelled terminals can establish an excitatory, asymmetric synapse with one dendrite and an inhibitory, symmetric synapse with another dendrite, raises the possibility that these terminals evoke different effects through different dendrites. Coexistence of ChAT and GABA has been observed in neurons in several areas of the central nervous system. These neurons include amacrine cells in the retina, hypoglossal motoneurons, dorsal horn cells in the spinal cord, and interneurons in the cerebral cortex $[7,13]$. A clue to the possible interactions between ChAT and GABA profiles in the DC might be inferred from the retinal amacrine cells, which can also be single ChAT-, single GABA-, and double ChAT/GABA-labelled. In the rabbit retina the wiring of the GABAergic and cholinergic amacrine neurites determines the outcome of computations creating directional sensitivity [2]. Since neurons in the DC may perform similar mathematical functions [22], they may operate with similar cellular mechanisms.

The source of the cholinergic terminals involved in crest synapses eludes us. The present demonstration that such crest synapses exist in the DC suggests that the NPH is one of the sources. However, the fact that we have not been able to trace these types of terminals from the NPH (see also [10]) and the finding that the cholinergic input to the DC is only partially depleted after lesions of the NPH [4], leaves open the possibility that terminals forming crest synapses are derived from another origin. Crest synapses in the DC and VLO have been traced from the ventral dentate nucleus and dorsal group y [8], but cholinergic neurons have not been observed in these nuclei, neither in the present nor in a previous study [25].

\section{Acknowledgements}

The excellent technical assistance of Eddie Dalm, Hans van der Burg, and Erika Goedknegt is greatly appreciated. The authors are also grateful to Profs. R.M. Buijs
(Amsterdam, Netherlands), C. Cozzari (Rome, Italy), and E. Mugnaini (Chicago, USA) for providing the antibodies.

\section{References}

[1] Alley, K., Baker, R. and Simpson, J.I., Afferents to the vestibulocerebellum and the origin of the visual climbing fibres in the rabbit. Brain Res., 98 (1975) 582-589.

[2] Ariel, M. and Daw, N.W., Pharmacological analysis of directional sensitive rabbit retinal ganglion cells, J. Physiol. 324 (1982) 161185.

[3] Azizi, S.A. and Woodward, D.J.. Inferior olivary complex of the rat: morphology and comments on the principles of organization within the olivocerebellar system, J. Comp. Neurol., 263 (1987) 467-484.

[4] Barmack. N.H., Fagerson, M. and Errico. P.. Cholinergic projection to the dorsal cap of the inferior olive of the rat, rabbit, and monkey. J. Comp. Neurol., 328 (1993) 263-281.

[5] Barmack, N.H. and Simpson, J.I.. Effects of microlesions of dorsal cap of inferior olive of rabbits on optokinetic and vestibuloocular reflexes, J. Neurophysiol., 43 (1980) 182-206.

[6] Buijs, R.M., van Vulpen, E.H.S. and Geffard. M., Ultrastructural localization of GABA in the supraoptic nucleus and the neural lobe. Neuroscience, 20 (1987) 347-355.

[7] Davidoff, M.S. and Schulze, W., Coexistence of GABA- and choline acetyltransferase (ChAT)-like immunoreactivity in the hypoglossal nucleus of the rat. Histochemistry, 89 (1988) 25-33.

[8] De Zeeuw. C.I., Gerrits, N.M., Voogd, J., Leonard. C.S. and Simp son, J.I., The rostral dorsal cap and ventrolateral outgrowth of the rabbit inferior olive receive a GABAergic input from dorsal group y and the ventral dentate nucleus, J. Comp. Neurol. 341 (1994) $420-432$

[9] De Zeeuw, C.I., Holstege, J.C., Ruigrok, T.J.H. and Voogd, J., Ultrastructural study of the GABAergic, cerebellar, and mesodiencephalic innervation of the cal medial accessory olive: anterograde tracing combined with immunocytochemistry. I. Comp. Neurol., $284(1989) \quad 12-35$

[10] De Zeeuw, C.I., Wentzel, P. and Mugnaini. E., Fine structure of the dorsal cap of the inferior olive and its GABAergic and nonGABAergic input from the nucleus prepositus hypoglossi in rat and rabbit. J. Comp. Neurol., 327 (1993) 6.3-82.

[11] Hess. D.T., The tecto-olivary-cerebellar pathway in the rat. Brain Res.. 250 (1982) 143-148.

[12] Ito. M., Nisimaru. N. and Yamamoto, M., Specific patterns of neuronal connections involved in the control of the rabbit's vestibulo-ocular reflexes by the cerebellar flocculus, $J$. Phssiol. Lond. 265 (1977) 838-854.

[13] Kosaka. T.. Tauchi, M. and Dahl, J.L., Cholinergic neurons containing GABA-like and/or glutamic acid decarboxylase-like immunoreactivities in various brain regions of the rat. Exp. Brain Res., 70 (1988) 605-617.

[14] Maekawa. K. and Takeda, T., Origin of descending afferents to the rostral part of dorsal cap of inferior olive which transfers contralateral optic activities to the flocculus. A horseradish peroxidase study, Brain Res, 172 (1979) 393-405.

[15] Mizuno. N.. Nakamura, Y. and Iwahori. N.. An electron microscope study of the dorsal cap of the inferior olive in the rabbit, with special reference to the pretecto-olivary fibers. Brain Res. 77 (1974) 385395.

[16] Mugnaini. E. and Oertel, W.H., An atlas of the distribution of GABAergic neurons and terminals in the rat CNS as revealed by GAD-immunohistochemistry. In A. Björklund and T. Hökfelt (Eds.). GABA and Neuropeptides in the CNS. The Handbook of Chemical Neuroanatomy, Vol. 4, Part I. Elsevier. Amsterdam, 1985, pp. $436-622$. 
[17] Nelson, B.J. and Mugnaini, E., The rat inferior olive as seen with immunostaining for glutamate decarboxylase, Anat. Embryol., 179 (1988) $109-127$.

[18] Nunes Cardozo, J.J. and van der Want, J.J.L., Ultrastructural organization of the retino-pretecto-olivary pathway in the rabbit: a combined WGA-HRP tracing and GABA immunocytochemical study, $J$. Comp. Neurol., 291 (1990) 313-327.

[19] Oertel, W.H., Schmechel, D.E., Tappaz, M.L. and Kopin, I.J., Production of a specific antiserum to rat brain glutamic acid decarboxylase by injection of an antigen-antibody complex, Neuroscience, 6 (1981) 2689-2700.

[20] Ruigrok, T.J.H., Osse, R.-J. and Voogd, J., Organization of inferior olivary projections to the flocculus and ventral paraflocculus of the rat cerebellum, J. Comp. Neurol., 316 (1992) 129-150.

[21] Seguela, P., Geffard, M., Buijs, R. and Le Moal, M., Antibodies against gamma-aminobutyric acid: specificity studies and immunocytochemical results, Proc. Natl. Acad. Sci. USA, 81 (1984) 38883892.
[22] Simpson, J.I. and Alley, K.E., Visual climbing fiber input to rabbit vestibulo-cerebellum: a source of direction-specific information, Brain Res., 82 (1974) 302-308.

[23] Sotelo, C., Gotow, T. and wassef, M., Localization of glutamic-acid decarboxylase-immunoreactive axon terminals in the inferior olive of the rat, with special emphasis on anatomical relations between GABAergic synapses and dendrodendritic gap junctions, $J$. Comp. Neurol. 252 (1986) 32-50.

[24] Umbriaco, D., Watkins, K.C., Descarries, L., Cozzari, C. and Hartman, B.K., Ultrastructure and morphometric features of the acetylcholine innervation in adult rat parietal cortex: an electron microscopic study in serial sections, J. Comp. Neurol., 348 (1994) 351-373.

[25] Woolf, N.J., Cholinergic systems in mammalian brain and spinal cord. Prog. Neurobiol, 37 (1991) 475-524. 